\title{
A Research on Dissipative Structure of the Information Resource Organization of E-Government
}

\author{
Na Wang ${ }^{1}$, Wenyi Zhang ${ }^{2}$, Xiaofang Wang ${ }^{3}$ and Lu Liu ${ }^{3}$ \\ ${ }^{1}$ School of Economics and Management, Beihang University, Beijing 100083, P.R. China \\ seaskyinter@163.com liulu@buaa.edu.cn \\ ${ }^{2}$ School of Computer Science and Technology, Harbin Engineering University, Harbin \\ 150001, P.R. China if3699@vip.163.com \\ ${ }^{3}$ Department of Mathematics, Jilin University, Changchun 130012, P.R. China \\ wang_xiaofeng80@163.com
}

\begin{abstract}
Government information resource is the foundation of e-government, and government information resource organization pattern is the key factor that affects sustainable development of e-government. At present, a lack of the systematic plan in government information resource organization exists universally. The primary reason is that systematization and formalization of research on government information resource organization pattern is insufficient and lacks support to organize and plan the government information resource. This article starts with the basic assumption of the government information hyper-cycle system consisting of e-government and its object system, depicts the dissipative structure of government information resource organization through introducing superior-rank operator and lower-rank operator in algebra system, and explores actively the self-organized, extensible and universal government information resource organization pattern based on hyper-cycle (PBHC) systematic plan.
\end{abstract}

Keywords: e-Government, Information resource, Dissipative structure, Selforganization

\section{INTRODUCTION}

In the primary tendency of the global informationization, e-government becomes gradually the important means to improve the level of international public administrative management and to enhance national competitive power, and more and more nations and governments attach importance to the plan and construction of egovernment. A mass of practices indicated that the development of e-government has the remarkable self-organizing characteristics, which is an important composing part of the human society evolvement from the industry society to the information society. Therefore, to research government information resource organization pattern, and to form the self-organization mechanism to support the sustainable development of egovernment, has been already the urgent duty in the plan and construction of egovernment.

Please use the following format when citing this chapter:

Wang, N., Zhang, W., Wang, X., Liu, L., 2007, in IFIP International Federation for Information Processing, Volume 255, Research and Practical Issues of Enterprise Information Systems II Volume 2, eds. L. Xu, Tjoa A., Chaudhry S. (Boston: Springer), pp. 1449-1455. 
This article regards the government operations as many kinds of catalysis cycle effecting upon the reaction cycle of civil economy and society development, and assume that e-government and its object system form a government information hyper-cycle system [1], and forms a kind of government information resource organization pattern by research on the dissipative structure of government information resource which is the basic conditions to form self-organized system, and based on constructing rank algebra system to prove the order and the sustainable development of this pattern structure.

\section{RANK ALGEBRA}

In order to study the order and the sustainable development of the government information resource organization, we firstly establish rank algebra $(R A)$ and its elementary theory. $R A$ is an algebra system that regards rank function satisfying some algebraic law as elementary operation.

Suppose $F$ is a mapping set, in which each mapping $f$ has non-negative integer $\mathbf{n}$ (to be called the rank of $f$ ). $F_{n} \in F$ is n mapping subset. $F_{0}$ is called constant set.

Definition 1.1 Suppose $A=\left\{X^{1}, X^{2}, X^{3}, \ldots, X^{m}\right\}$ is a non-empty set, $X^{r}, r=1,2, \ldots, m$ is called class-member of $A$, if

1. There are constants $a_{1}^{X^{r}}, a_{2}^{X^{r}}, \ldots$ in $X^{r}$

2. There are variables $y_{1}^{X^{r}}, y_{2}^{X^{r}}, \ldots$ in $X^{r}$

3. There are mappings $f$ in $X^{r}$

$A$ is called a non-empty class-member set. All of the constants, variables and mappings in $X^{r}$ are called the members of $X^{r}$.

Definition 1.2 Suppose $A$ is a non-empty class-member set, $\langle A, \leq\rangle$ with a partial order " $\leq$ "is called a partially ordered class-member set.

Definition 1.3 Let $X^{i}, X^{j} \in A$, if $X^{i} \leq X^{j}$ or $X^{j} \leq X^{i}$ hold, then $X^{i}$ and $X^{j}$ are comparable.

Definition 1.4 Suppose $\langle A, \leq\rangle$ is a non-empty partially ordered class-member set, $X^{i}$ and $X^{j}$ are the class-members of $\langle A, \leq\rangle$, if $X^{i} \leq X^{j}$, then $X^{j}$ has a higher rank than $X^{i}$. If $X^{i} \leq X^{j}$ and $X^{j} \leq X^{i}$ hold at the same time, then $X^{i}$ and $X^{j}$ have equivalent rank, denoted by $X^{i} \hat{=} X^{j}$.

The set composed of all of the equivalent-rank class-members is called equivalentrank class-members set $\langle\Theta, \hat{=} \operatorname{rank}(\Theta)>$, where $\operatorname{rank}(\Theta)$ is the rank. Simply, $<\Theta, \stackrel{\wedge}{=} \operatorname{rank}(\Theta)>$ is denoted by $\Theta$.

Definition 1.5 Suppose $\Theta$ is a equivalent-rank class-members set of $\langle A, \leq\rangle$, $X^{i}$ and $X^{j}$ are the class-members in $\Theta$, for all $x^{i} \in X^{i}$ and $x^{j} \in X^{j}, x^{i}$ and $x^{j}$ have a equivalent rank, denoted by $x^{i} \stackrel{\wedge}{=} x^{j}$. All of the members which have an equivalent rank in $\Theta$ compose an equivalent-rank member set, denoted by $\Pi$. 
Definition 1.6 Suppose $\Theta^{\prime}$ and $\Theta^{2}$ are equivalent-rank class-member sets of $\langle A, \leq\rangle$, $\Pi^{1}$ and $\Pi^{2}$ are respectively equivalent-rank class-member sets of $\Theta^{1}$ and $\Theta^{2}$, and $\Pi_{1}$ and $\Pi_{2}$ are respectively sub-sets of $\Pi^{1}$ and $\Pi^{2}, \Pi_{1}$ and $\Pi_{2}$ are respectively called equivalent-rank member subset of $\Theta^{1}$ and $\Theta^{2}$.If there exist $x^{i} \in \Pi_{1}, x^{j} \in \Pi_{2}$, and $x^{i} \prec x^{j}$, then $\Pi_{2}$ is a higher equivalent-rank member subset, denoted by $\Pi_{1} \prec \Pi_{2}$.

Definition 1.7 Suppose $\Theta^{1}$ and $\Theta^{2}$ are equivalent-rank class-member sets of $\langle A, \leq\rangle$, $\Pi_{1}$ and $\Pi_{2}$ are respectively equivalent-rank member subsets of $\Theta^{1}$ and $\Theta^{2}, \Pi_{1} \prec \Pi_{2}$ $\varphi^{\Pi_{1}}: \Pi_{1} \mid \rightarrow \Pi_{2}, \varphi \in F$, then $\varphi^{\Pi_{1}}$ is called a superior-rank operator.

The rank of the superior-rank operator $\varphi^{\Pi_{1}}$ is denoted by rank $\left(\varphi^{\Pi_{1}}\right)=$ rank $\left(\Pi_{2}\right)$-rank $\left(\Pi_{1}\right)>0$. The set composed of all the superior-rank operators is called a superior-rank operator set, denoted by $\Phi$.

Definition 1.8 Suppose $\Theta^{\mathrm{l}}$ and $\Theta^{2}$ are equivalent-rank class-member sets of $\langle A, \leq\rangle$, $\Pi_{1}$ and $\Pi_{2}$ are respectively equivalent-rank member subsets of $\Theta^{1}$ and $\Theta^{2}, \Pi_{1} \prec \Pi_{2}$ $\mu^{\Pi_{2}}: \Pi_{2} \mapsto \Pi_{1}, \mu \in F$, then $\mu^{\Pi_{2}}$ is called a lower-rank operator.

The set composed of all the lower -rank operators is called a lower-rank operator set, denoted by $\Psi$.

Definition 1.9 Suppose $\Theta$ is an equivalent-rank class-member set of $\langle A, \leq\rangle, \Pi_{1}$ and $\Pi_{2}$ are respectively equivalent-rank member sub-sets of $\Theta$

$\eta^{\Pi_{1}}: \Pi_{1} \mid \rightarrow \Pi_{2}, \quad \eta \in F$, then $\eta^{\Pi_{1}}$ is called an even-rank operator.

The set composed of all the even-rank operators is called an even-rank operator set, denoted by $\aleph . \lambda^{i}=\Phi^{i} \cup \Psi^{i} \cup \aleph^{i}$ is called $i$ th class rank operator set.

Definition 1.10 Let $H=\left\{\lambda^{1}, \lambda^{2}, \ldots, \hbar^{N}\right\}, \lambda^{i}, i=1,2, \ldots, N$ is $i$ th class rank operator set, then $H$ is the set of all class rank operators.

Definition 1.11 Suppose $\langle A, \leq\rangle$ is a non-empty partially ordered class-member set, $X^{r}$ is a class-member of $\langle A, \leq\rangle, H=\left\{\lambda^{1}, \lambda^{2}, \ldots, \lambda^{N}\right\}$ is the set of all class rank operators, if for $\forall \xi \in X^{r}, \quad 1 \leq r \leq m$, there exists $\gamma^{i} \in \lambda^{i}$, such that $\gamma^{i}(\xi) \in X^{j} \in A, 1 \leq j \leq m$, then $H$ is closed in $\langle A, \leq\rangle$.

Definition 1.12 Suppose $\langle A, \leq\rangle$ is a non-empty partially ordered class-member set, $H$ is the closed set of all class rank operators, then $\langle A, H\rangle$ is an algebraic system which is called $R A$ (rank algebra).

Lemma 1.1 If $\mu \circ \eta$ is meaningful, $\operatorname{rank}(\mu \circ \eta)=\operatorname{rank}(\mu)+\operatorname{rank}(\eta)$ 。 


\section{EVOLUTION RULE of RANK ALGEBRA}

Based on rank algebra in second section, we study the evolution rule about the order and the sustainable development in rank algebra.

Definition 2.1 Suppose $\Theta^{1}$ and $\Theta^{2}$ are equivalent-rank class-member sets of $\langle A, \leq\rangle$, $\Pi_{1}$ and $\Pi_{2}$ are respectively equivalent-rank member subsets, $\Pi_{1} \prec \Pi_{2}$, if there exists $h: \Pi_{1} \mapsto \Pi_{2}$ or $h: \Pi_{2} \mapsto \Pi_{1}$, where $h \in \lambda^{i} \in H=\left\{\lambda^{1}, \lambda^{2}, \ldots, \lambda^{N}\right\}$, then $\operatorname{rank}\left(\Pi_{2}\right)$ $\operatorname{rank}\left(\Pi_{1}\right)$ is the order $\Pi_{1}$ to $\Pi_{2}$, denoted by $\operatorname{order}\left(\Pi_{1}, \Pi_{2}\right)$.

Lemma 2.1 Suppose $\Theta^{\prime}$ and $\Theta^{2}$ are equivalent-rank class-member sets of $\langle A, \leq\rangle$, $\Pi_{1}$ and $\Pi_{2}$ are respectively equivalent-rank member subsets, for arbitrary $x_{1} \in \Pi_{1}$ and $x_{2} \in \Pi_{2}, \operatorname{order}\left(x_{1}, x_{2}\right)=\operatorname{order}\left(\Pi_{1}, \Pi_{2}\right)$.

Theorem 2.1 Suppose that $\langle A, H\rangle$ is rank algebra, then $\langle A, H\rangle$ certainly evolve and develop from the lower ordered state to the higher ordered state.

\section{DISSIPATIVE STRUCTURE OF GOVERNMENT INFORMATION RESOURCE ORGANIZATION}

In this section, we firstly establish government information resource organization pattern based on hyper-cycle and research the dissipative structure evolvement rule of the government information resource organization.

\subsection{Government Information Resource Organization Pattern Based on Hyper-cycle-PBHC}

Suppose that e-government and its object system compose government information hyper-cycle system ${ }^{[8]}, A$ is the information resource set of government object system, and $B$ is the information resource set of government activity, if government information resource set $G=A \cup A \times A \cup B \cup A \times B \cup B \times B$ satisfies the (1) (B) conditions, and the class-member of the higher-rank information class-member set and the class-member of the lower-rank information class-member set compose the new information class-member which has an equivalent rank with the class-member of the higher-rank information class-member set, then $G$ is called government information resource organization pattern based on hyper-cycle which is denoted by PBHC for simply.

(1) $A=\left\{X_{1}^{0}, X_{2}^{0}, \ldots, X_{L}^{0}, X_{1}^{1}, X_{2}^{1}, \ldots, X_{M}^{1}, X_{1}^{2}, X_{2}^{2}, \ldots, X_{N}^{2}, \ldots ., X_{1}^{P}, X_{2}^{P}, \ldots, X_{Q}^{P}\right\}$ is a non-empty partially ordered class-member set, and $C^{0}=A^{0}=\left\{X_{1}^{0}, X_{2}^{0}, \ldots, X_{6}^{0}\right\}$ denotes the information resource set of the social basic essential factors such as government organization, population, legal person organization, natural resources, environment, information and so on. 
$C^{1}=A^{1}=\left\{X_{1}^{1}, X_{2}^{1}, \ldots, X_{5}^{1}\right\}$ denotes the information resource set of the essential factors of production and circulation such as product, production, currency, consumption, money and so on. $C^{2}=A^{2}=\left\{X_{1}^{2}, X_{2}^{2}, \ldots, X_{6}^{2}\right\}$ denotes the information resource set of the essential factors of the value increment activity such as finance, science and technology, credit, promulgating, sanitation, amusement and so on. $C^{3}=A^{3}=\left\{X_{1}^{3}, X_{2}^{3}, \ldots, X_{5}^{3}\right\}$ denotes the information resource set of the essential factors of the mechanism of maintaining the society such as law, morality, civilization, education, security and so on. The rank $(0 \sim 3)$ of the four information resource sets $C^{0}, C^{1}, C^{2}, C^{3}$ and their mapping to the e-government object system depict the rank and the rule of the internal reaction cycle of the e-government object system organization. In other words, the social basic constitution, the activity of production and circulation, value increment activity and the mechanism of maintaining the society denote the rank and the evolution rule of the society from the lover-order state to the higher-order state.

(2) $A \times A=\left(Z_{p q}^{i j}\right)_{22 \times 22}$ is sparse matrix, where $Z_{p q}^{i j}=X_{p}^{i} \circ X_{q}^{j}$ denotes composite information class-member of $X_{p}^{i}$ and $X_{q}^{j}, Z_{p q}^{i j}=X_{p}^{i} \circ X_{q}^{j} \in G$ only when composite information class-member $Z_{p q}^{i j}$ has precise physical meaning. We denote that $C^{19}=A^{0} \times A^{0}, C^{20}=A^{0} \times A^{1}, \quad C^{21}=A^{0} \times A^{2}, \quad C^{22}=A^{0} \times A^{3}, \quad C^{23}=A^{1} \times A^{1}$, $C^{24}=A^{1} \times A^{2}, \quad C^{25}=A^{1} \times A^{3}, \quad C^{26}=A^{2} \times A^{2}, \quad C^{27}=A^{2} \times A^{3}, \quad C^{28}=A^{3} \times A^{3}$.

(3) $B=\left\{Y_{1}^{1}, Y_{2}^{1}, Y_{1}^{2}, Y_{2}^{2}, Y_{1}^{3}, Y_{2}^{3}\right\}$ is a non-empty partially ordered class-member set, $Y_{1}^{\mathrm{1}}$ is the information about government monitoring information, corresponding to rank operator set $\lambda^{1}$ of monitoring information; $Y_{2}^{1}$ is the information about government enforcing laws and statutes, corresponding to rank operator set $\lambda^{2}$ of enforcing laws and statutes; $Y_{1}^{2}$ is the information about government financial revenue and expenditure, corresponding to rank operator set $\lambda^{3}$ of financial revenue and expenditure; $Y_{2}^{2}$ is the information about government self-management, corresponding to rank operator set $\lambda^{4}$ of self-management; $Y_{1}^{3}$ is the information about government macroscopic decision, corresponding to rank operator set $\lambda^{5}$ of macroscopical decision; $Y_{2}^{3}$ is the information about government dealing with emergency, corresponding to rank operator set $\lambda^{6}$ of dealing with emergency. Let the operator set $H=\left\{\lambda^{1}, \lambda^{2}, \lambda^{3}, \lambda^{4}, \lambda^{5}, \lambda^{6}\right\}$ be the e-government system. The rank $(4 \sim 6)$ of the three information resource

sets $C^{4}=B^{1}=\left\{Y_{1}^{1}, Y_{2}^{1}\right\}, C^{5}=B^{2}=\left\{Y_{1}^{2}, Y_{2}^{2}\right\}, C^{6}=B^{3}=\left\{Y_{1}^{3}, Y_{2}^{3}\right\}$ depicts the rank of the egovernment system. Based on the general cybernetics, the e-government system can form a kind two-level control structure that has the source and the handling unconventionality mechanism.

(4) $A \times B=\left(R_{p q}^{i j}\right)_{22 \times 6}$ is $22 \times 6$ matrix, where $R_{p q}^{i j}=X_{p}^{i} \circ Y_{q}^{j}$ denotes composite information class-member of $X_{p}^{i}$ and $Y_{q}^{j}, R_{p q}^{i j}=X_{p}^{i} \circ Y_{4}^{j} \in G$ only when composite 
information class-member $R_{p q}^{i j}$ has precise physical meaning. We denote that $C^{7}=A^{0} \times B^{1}, \quad C^{8}=A^{1} \times B^{1}, \quad C^{9}=A^{2} \times B^{1}, \quad C^{10}=A^{3} \times B^{1}, \quad C^{11}=A^{0} \times B^{2}$, , $C^{12}=A^{1} \times B^{2}, \quad C^{13}=A^{2} \times B^{2}, \quad C^{14}=A^{3} \times B^{2}, \quad C^{15}=A^{0} \times B^{3}, \quad C^{16}=A^{1} \times B^{3}$, $C^{17}=A^{2} \times B^{3}, \quad C^{18}=A^{3} \times B^{3}$.

(5) $B \times B=\left(S_{p q}^{i j}\right)_{6 \times 6}$ is $6 \times 6$ matrix, where $S_{p q}^{i j}=Y_{p}^{i} \circ Y_{q}^{j}$ denotes composite information class-member of $Y_{p}^{i}$ and $Y_{q}^{j}, S_{p q}^{i j}=Y_{p}^{i} \circ Y_{q}^{j} \in G$ only when composite information classmember $S_{p q}^{i j}$ has precise physical meaning. We denote that $C^{29}=B^{1} \times B^{1}$, $C^{30}=B^{1} \times B^{2}, \quad C^{31}=B^{1} \times B^{3}, \quad C^{32}=B^{2} \times B^{2}, \quad C^{33}=B^{2} \times B^{3}=B^{3} \times B^{2}$,
$C^{34}=B^{3} \times B^{3}$.

(6) The even-rank class-member sets $C^{0} \sim C^{34}$ satisfy the partial order as follow.

$$
\begin{aligned}
& C^{0} \triangleq C^{19} \prec C^{1} \hat{=} C^{20} \hat{=} C^{23} \prec C^{2} \hat{=} C^{21} \hat{=} C^{24} \prec C^{3} \hat{=} C^{26} \hat{=} C^{22} \hat{=} C^{25} \hat{=} C^{27} \hat{=} C^{28} \prec \\
& C^{4} \triangleq C^{7} \triangleq C^{8} \triangleq C^{9}=C^{10} \prec C^{5} \hat{=} C^{11} \hat{=} C^{12} \hat{=} C^{13} \hat{=} C^{14} \prec C^{6} \hat{=} C^{15} \hat{=} C^{16} \hat{=} C^{17} \hat{=} C^{18} \\
& C^{29} \triangleq C^{30} \triangleq C^{31} \prec C^{32} \triangleq C^{33} \prec C^{34}
\end{aligned}
$$

\subsection{Evolution Rule of PBHC to Dissipative Structure}

We prove that PBHC has four conditions to form and maintain dissipative structure [2] [3] as follows.

Lemma 3.1 Government information resource organization pattern based on hypercycle (PBHC) $G=A \cup A \times A \cup B \cup A \times B \cup B \times B$ is an open system.

Lemma 3.2 Government information resource organization pattern based on hypercycle (PBHC) $G=A \cup A \times A \cup B \cup A \times B \cup B \times B$ must evolve and develop to nonlinear far away the equilibrium state.

Lemma 3.3 There must be certain non-linear dynamics process in government information resource organization pattern based on hyper-cycle (PBHC) $G=A \cup A \times A \cup B \cup A \times B \cup B \times B$.

Lemma 3.4 Government information resource organization pattern based on hypercycle (PBHC) $G=A \cup A \times A \cup B \cup A \times B \cup B \times B$ forms new order through fluctuation.

According to lemma 3.1 to lemma 3.4, and theorem2.1, government information resource organization pattern based on hyper-cycle (PBHC) $G=A \cup A \times A \cup B \cup A \times B \cup B \times B$ will certainly evolve and develop from the lower ordered state to the higher ordered state.

\section{CONCLUSIONS}

This article regards government information resource set $G$ as a partially ordered class-member set, and defines equivalent-rank class-members set and partial order on $G$, and further defines the catalysis mechanism that government activity acts on the reaction cycle of the object system as rank operator set acting on $G$; Thus, while 
A Research on Dissipative Structure of the Information Resource Organization of E-

Government 1455

defining government information resource organization pattern PBHC based on hyper-cycle, and make it conform to the elementary condition of constructing rank algebra. Finally, in virtue of the rank operator property of government activity, this article proves that $\mathrm{PBHC}$ is the self-organizing dissipative structure. And PBHC as the theory support of developing and using the government information resource task plan is applied widely in " $11^{\text {th }}$ five-year" plan of national e-government.

\section{REFERENCES}

1. W.Y. Zhang, Research on informationization theories, material collection of important subject research of the National Development and Innovation Committee, The 11th fiveyear informationization special project plan (2005).

2. R. Melamede, Dissipative Structures and the Origins of Life. http://www.uccs.edu/ rmelamed/Papers/NECSI.htm(Accessed July 14, 2007)

3. T. Wu, Research on Self-organization Methodology (Tsinghua University Publishing House: Beijing, 2001). 